\title{
Effects of Sargassum horneri and Ulva australis Extracts on Body Weight and Serum Glucose Levels of Sprague-Dawley Rats
}

\author{
Young-Hyeon Lee ${ }^{1}$, Min-Ho Yeo ${ }^{1}$, Seon-A Yoon ${ }^{2}$, Ho-Bong Hyun ${ }^{2}$, Young-Min Ham², Yong-Hwan Jung ${ }^{2}$, \\ and Kyung-Soo Chang ${ }^{1}$
}

${ }^{1}$ Department of Clinical Laboratory Science, Catholic University of Pusan, Busan 46252, Korea

${ }^{2}$ Biodiversity Research Institute, Jeju Technopark, Jeju 63608, Korea

\begin{abstract}
This study investigated the safety and functionality of a functional additive for humans and animals from Sargassum horneri (SH) and Ulva australis (UA) waste for recycling marine refuse generated in large quantities in Jeju. SpragueDawley rats were orally administered functional additives at $2,000 \mathrm{mg} / \mathrm{kg}$ to assess 14-day repeated dose toxicity of the two extracts. For female rats, weight gain after administration of SH was $66.2 \pm 18.8 \%$ vs. controls. Male rats administered UA showed weight gain of $92.3 \pm 8.0 \%$ vs. controls. SH and UA significantly decreased serum glucose levels in male rats compared with controls $(79.8 \pm 11.10 \%$ and $76.1 \pm 9.67 \%$, respectively). Similarly, significant decrease in serum glucose levels were shown for female rats after administration of SH and UA $(79.2 \pm 1.58 \%$ and $82.8 \pm 3.21 \%$, respectively). Furthermore, rats showed significant differences vs. controls in several serological parameters after receiving extracts, however results remained within the normal range. Thus, the SH and UA extracts were considered safe substances that may be used as functional additives to help reduce body weight and serum glucose.
\end{abstract}

Keywords: functional natural additive, Sargassum horneri, safety evaluation, serum glucose, Ulva australis

\section{INTRODUCTION}

Marine waste [e.g., from Sargassum horneri (SH) and Ulva australis (UA)] is generated in large quantities in the Jeju region, where it is recognized as an environmental pollutant and an obstacle to achieving a clean environment. Before 2013, organic waste resources were disposed of by dumping in the sea. However, such disposal has since been prohibited by the London Convention Protocol. Wastes are now disposed in landfills or eliminated by incineration. Limitations to these methods encourage the development of other options, including suppressing waste generation and developing an appropriate recycling method (Ahn et al., 2007; Kim et al., 2007; Kim et al., 2014).

$\mathrm{SH}$ is a yellowish-brown species of alga with thin and spatula-shaped leaves, which grows mainly during winter. $\mathrm{SH}$ is rich in dietary fiber, polysaccharides, amino acids, and vitamins, and is considered a delicacy in East Asia (Matsumura, 2001; Dias et al., 2020). However, the thallus of $\mathrm{SH}$, which is increasing due to $\mathrm{SH}$ aquaculture in the waters of Zhejiang Province, China, is carried away from substrates and down prevailing currents (Preeprame et al., 2001; Liu et al., 2012; Kim, 2015). SH is now widely distributed throughout the east and south coasts of Korea and along all of the Japanese coastline. Indeed, an average of 300 9,000 tons of SH flowed into Jeju Island from 2015 to 2017 . The giant seaweed grows with the help of microorganisms, provides a haven for reproduction of many marine species, and supplies nutrients to herbivorous marine life (Kim et al., 2018a). Still, the inflow of thallus causes environmental and economic problems, such as fouling fishing boat screws, impeding fishing and sailing, becoming trapped in and damaging aquaculture facilities, and causing odors due to stranding of thallus on shorelines (Byeon et al., 2019; Kim et al., 2019). Previous studies have demonstrated the ability of $\mathrm{SH}$ in prevention of cardiovascular disease (Cardoso et al., 2015), inhibition of atopy (Kim et al., 2020), inhibition of adipocyte production (Kwon et al., 2019), inhibition of cancer cell growth (Choi et al., 2006), and anticoagulation (Kim et al., 2004).

UA is a primary cause of coastal pollution in Jeju, where it grows singly or in groups of two or three (Lin et al., 
2011). UA becomes established in a wide range of temperatures and salinities, and displays various reproductive strategies and rapid growth (Zhang et al., 2013). UA grows from April to August in areas with flowing freshwater flows, along the coast of Jeju. Since UA reproduces in groups, it interferes with marine habitats and other algae (Kim et al., 2018b). Furthermore, UA occurs in large quantities at major tourist destinations, resulting in loss of recreational beach use. The seaweed may also cause a stench due to decomposition along shorelines, causing a nuisance to nearby residents. UA is also distributed in the Mediterranean, Pacific, and Indian Oceans, and is a food rich in dietary fiber, protein, and minerals (Chi et al., 2020). In addition, UA is used in traditional Chinese medicine to treat hyperlipidemia, heatstroke, and urinary diseases (Qi et al., 2012). Moreover, UA displays many polysaccharide-containing sulfate groups and has been reported to exhibit antitumor, anti-hyperlipidemia, and immunomodulatory activity (Patel, 2012; Song et al., 2015). However, only a fraction of UA is used in foods and medications, with the remainder disposed of as waste.

Various studies are ongoing to determine how SH and UA can be safely recycled without causing adverse environmental impact. For example, people are actively pursuing extracting active ingredients from algal by-products for bioenergy production, pulp manufacturing, and fucoidan. However, research on the safety and utility of the waste as food additives, which may efficiently utilize marine organic wastes, or as functional additives for human consumption, is currently insufficient (Hong et al., 2011). Therefore, this study examined the safety and functionality of additives from $\mathrm{SH}$ and UA for use by humans and animals. We assessed the clinical symptoms, weight changes, and blood and serological changes of SpragueDawley rats following oral administration of $\mathrm{SH}$ and $\mathrm{UA}$ waste extracts.

\section{MATERIALS AND METHODS}

\section{Extract preparation}

SH and UA collected from the coast of Jeju Island were washed and dried, then pulverized with a grinder and extracted. Fifty grams of dried samples were immersed in $1 \mathrm{~L}$ of $80 \%$ ethanol and stirred for $24 \mathrm{~h}$. The ethanol suspension was filtered using a $0.45 \mu \mathrm{m}$ bottle filter (Corning Bottle-Top Vacuum Filter System, Corning Inc., Corning, NY, USA), and the filtrate lyophilized after evaporating the ethanol using a vacuum concentrator to prepare a powder. The powder was stored at $-20^{\circ} \mathrm{C}$ and used as a suspension in sterile distilled water.

\section{Animals and animal experiments}

Five-week-old female and male Sprague-Dawley rats housed in specific-pathogen free conditions were purchased from KOSA BIO Inc. (Gyeonggi, Korea). After one week of quarantine and acclimatization, healthy animals were used in experiments. Animals were kept at $23 \pm 3^{\circ} \mathrm{C}$, a relative humidity of $50 \pm 10 \%$, and with $12 \mathrm{~h}$ of illumination per day at 150 300 Lux. Rat feed (SAMTAKO BIO KOREA, Gyeonggi, Korea) and water were provided ad libitum. This research followed the Code of Ethics for Animal Experiments at Pusan Catholic University (CUP AEC 2020-002). Rats were divided into control, SH, and UA groups. Five female and five male animals were included in each group. The daily dose of extracts was $2,000 \mathrm{mg} / \mathrm{kg}$ administered in sterile water, the maximum concentration suggested by the Organization for Economic Co-operation and Development toxicity evaluation guidelines. Rats in the control group received sterile distilled water. Oral administration was continued for 14 days using a volume of $10 \mathrm{~mL} / \mathrm{kg}$.

\section{Clinical symptoms, body weight, and feed intake}

On the first day of oral administration of each substance, rats were observed from 1 to $6 \mathrm{~h}$. Throughout the study period, rats were observed for general symptomatic changes, toxic symptoms, decreased mobility, addiction, and death at designated times once daily. All laboratory rats were weighed every 2 days before and after extract administration, which measured feed intake at 3 4-day intervals.

\section{Necropsy and organ weight measurement}

Experimental rats were fasted $12 \mathrm{~h}$ before necropsy, using an anesthetic comprising of a mixture of alfaxalone (Alfaxan $^{\circledR}$; Careside Co., Ltd., Gyeonggi, Korea) $25 \mathrm{mg} /$ kg and xylazine (Rompun ${ }^{\mathbb{R}}$; Bayer Korea, Seoul, Korea) $5 \mathrm{mg} / \mathrm{kg}$. After anesthesia, blood was collected from the abdominal aorta, and gross organ abnormalities were recorded. Livers, lungs, hearts, kidneys, spleens, and thymuses were collected from all rats. In addition, ovaries and uteruses were collected from female rats, and testicles and prostates were collected from male rats. All organs were weighed after gross examination.

\section{Analysis of blood and serum biochemistry}

Blood was collected into ethylenediaminetetraacetic acid tubes (BD Caribe Ltd., Sumter, SC, USA), stirred on a roll mixer for $30 \mathrm{~min}$, and analyzed using a blood analyzer (DxH500, Beckman Coulter, Inc., Brea, CA, USA). White blood cell and red blood cell count (RBC), hemoglobin (HGB), hematocrit (HCT), mean red blood cell volume (MCV), mean corpuscular hemoglobin (MCH), mean corpuscular hemoglobin concentration (MCHC), and platelet (PLT) count were recorded.

Blood was collected into serum separator tube tubes (BD Caribe Ltd.) for serum biochemistry, allowed to co- 
agulate at room temperature, and centrifuged at 2,500 rpm for $20 \mathrm{~min}$. Serum obtained by centrifugation was analyzed using a blood biochemical analyzer BT1500 (Biotecnica Instruments SpA, Rome, Italy). Analyses included determination of total protein, albumin (ALB), aspartate aminotransferase (AST), alanine transaminase (ALT), gamma-glutamyl transferase, alkaline phosphatase (ALP), glucose (Glu), total cholesterol (CHO), triglyceride (TG), total bilirubin, high-density lipoprotein cholesterol (HDL-C), urea, and blood urea nitrogen (BUN) levels.

\section{Statistical analysis}

Data were analyzed using SPSS version 25 (Statistics Package for Social Sciences, Chicago, IL, USA), and expressed as mean \pm standard deviation (SD). Statistical analyses of body weight, feed intake, organ weight, blood, and serological values were performed using one-way ANOVA to compare multiple groups. Values with $P<0.05$ and $P<0.01$ were considered significantly different.

\section{RESULTS}

\section{General health and death observations}

After extract administration, there were no changes in clinical signs, such as salivation, reduced mobility, and toxicity, including in control rats. In addition, there were no drug-induced deaths among experimental or control rats (Table 1).

\section{Body weight and feed intake}

Body weight of both female and male rats increased from before to after extract administration. Male rats in the control group weighed $94.6 \pm 6.3 \mathrm{~g}$ after dosing compared with $90.8 \pm 6.8 \mathrm{~g}$ for rats in the $\mathrm{SH}$ group, and $87.3 \pm 7.5 \mathrm{~g}$ for rats in the UA group. Similar findings were observed for female rats: rats weighed $34.8 \pm 6.2 \mathrm{~g}, 23.0 \pm 6.6 \mathrm{~g}$, and $31.0 \pm 3.6 \mathrm{~g}$ in the control, $\mathrm{SH}$, and UA groups, respectively, after dosing (Table 2). Weight gain in male rats administered SH or UA was $96.0 \pm 7.2 \%$ and $92.3 \pm 8.0 \%$, respectively, of control rats. Weight gain following UA

Table 1. Clinical signs of Sprague-Dawley rats orally administered ethanol extracts of Sargassum horneri (SH) or U/va australis (UA) for 14 days

\begin{tabular}{|c|c|c|c|c|c|c|c|}
\hline \multirow[b]{2}{*}{ Extract } & \multirow[b]{2}{*}{ Sex } & \multirow[b]{2}{*}{$\begin{array}{c}\text { Dose } \\
\text { (mg/kg/d) }\end{array}$} & \multicolumn{5}{|c|}{ Signs observation } \\
\hline & & & $\begin{array}{l}\text { Decrease of } \\
\text { locomotor } \\
\text { activity }\end{array}$ & $\begin{array}{l}\text { Decreased } \\
\text { respiration } \\
\text { rate }\end{array}$ & Lacrimation & Salivation & Death \\
\hline \multirow[t]{4}{*}{$\mathrm{SH}$} & Male & 0 & $0 / 5^{1)}$ & $0 / 5$ & $0 / 5$ & $0 / 5$ & $0 / 5$ \\
\hline & & 2,000 & $0 / 5$ & $0 / 5$ & $0 / 5$ & $0 / 5$ & $0 / 5$ \\
\hline & Female & 0 & $0 / 5$ & $0 / 5$ & $0 / 5$ & $0 / 5$ & $0 / 5$ \\
\hline & & 2,000 & $0 / 5$ & $0 / 5$ & $0 / 5$ & $0 / 5$ & $0 / 5$ \\
\hline \multirow[t]{4}{*}{ UA } & Male & 0 & $0 / 5$ & $0 / 5$ & $0 / 5$ & $0 / 5$ & $0 / 5$ \\
\hline & & 2,000 & $0 / 5$ & $0 / 5$ & $0 / 5$ & $0 / 5$ & $0 / 5$ \\
\hline & Female & 0 & $0 / 5$ & $0 / 5$ & $0 / 5$ & $0 / 5$ & $0 / 5$ \\
\hline & & 2,000 & $0 / 5$ & $0 / 5$ & $0 / 5$ & $0 / 5$ & $0 / 5$ \\
\hline
\end{tabular}

${ }^{11}$ Number of animals with sign/total number of animals examined.

Table 2. Body weight changes of Sprague-Dawley rats orally administered ethanol extracts of Sargassum horneri (SH) or U/va australis (UA) for 14 days

\begin{tabular}{|c|c|c|c|c|c|c|}
\hline \multirow{3}{*}{ Day after treatment } & \multicolumn{3}{|c|}{ Male dose $(\mathrm{mg} / \mathrm{kg})$} & \multicolumn{3}{|c|}{ Female dose $(\mathrm{mg} / \mathrm{kg})$} \\
\hline & Control & $\mathrm{SH}$ & UA & Control & $\mathrm{SH}$ & UA \\
\hline & 0 & 2,000 & 2,000 & 0 & 2,000 & 2,000 \\
\hline 0 & $169.5 \pm 1.0$ & $170.5 \pm 3.2$ & $168.9 \pm 6.9$ & $146.8 \pm 4.8$ & $145.8 \pm 4.5$ & $147.7 \pm 2.7$ \\
\hline 2 & $190.2 \pm 4.2$ & $193.7 \pm 4.9$ & $190.8 \pm 7.7$ & $158.2 \pm 6.2$ & $151.8 \pm 4.7$ & $155.7 \pm 3.8$ \\
\hline 6 & $220.6 \pm 4.7$ & $223.8 \pm 3.5$ & $217.8 \pm 9.0$ & $171.3 \pm 4.5$ & $166.3 \pm 5.4$ & $168.7 \pm 5.9$ \\
\hline 10 & $253.2 \pm 5.3$ & $254.4 \pm 4.1$ & $249.3 \pm 8.4$ & $182.6 \pm 4.4$ & $181.4 \pm 5.3$ & $183.8 \pm 2.9$ \\
\hline 14 & $278.9 \pm 5.8$ & $276.0 \pm 2.5$ & $270.9 \pm 9.1$ & $192.2 \pm 5.7$ & $189.9 \pm 4.8$ & $192.1 \pm 4.0$ \\
\hline 15 & $264.1 \pm 6.7$ & $261.3 \pm 8.2$ & $256.2 \pm 10.1$ & $181.6 \pm 8.5$ & $168.9 \pm 7.9$ & $178.7 \pm 5.9$ \\
\hline Weight gain $(g)^{1)}$ & $94.6 \pm 6.3$ & $90.8 \pm 6.8$ & $87.3 \pm 7.5$ & $34.8 \pm 6.2$ & $23.0 \pm 6.6^{*}$ & $31.0 \pm 3.6$ \\
\hline Weight gain $(\%)^{2)}$ & $55.8 \pm 3.6$ & $53.2 \pm 3.9$ & $51.8 \pm 5.2$ & $23.7 \pm 4.1$ & $15.8 \pm 4.6^{*}$ & $20.9 \pm 2.1$ \\
\hline
\end{tabular}

Body weight values are expressed in grams (mean \pm SD).

Significant difference at ${ }^{*} P<0.05$ vs. the control group.

${ }^{11}$ Weight gain (g) differences between day 15 and day 0 .

${ }^{2)}$ Weight gain (\%) expressed as a percentage of the difference in weight between 15 and 0 days. 
administration was low, but not significantly different from that of controls (Fig. 1A). However, weight gain in female rats was $66.2 \pm 18.8 \%$ and $89.2 \pm 10.3 \%$ of controls after SH and UA administration, respectively. The difference in weight gain between female rats in the $\mathrm{SH}$ and control was statistically significant $(P<0.05)$ (Fig. 1B). Feed intake of female rats administered extracts tended to be lower than those of female control rat, however, feed intake did not differ significantly. Similar results were observed for male rats (Table 3). Since no differences in feed intake were seen, the algal extracts appeared to affect weight loss.

\section{Necropsy and organ weight measurements}

Necropsy of laboratory rats did not reveal any specific or abnormal findings associated with the extracts. Furthermore, there were no significant differences between control and experimental rats when all rats were grouped, or when rats were grouped by sex (Table 4 ).

\section{Analysis of blood and serum biochemistry}

Most hematological analyses for control and experimental rats were within normal ranges, with no significant alter- ations observed (Table 5). However, RBC in female rats increased from $6.32 \pm 0.77$ in the control group to $7.77 \pm$ 0.46 and $7.48 \pm 0.46$ in rats in the $\mathrm{SH}$ and UA groups, respectively. Furthermore, HGB levels were 13.44 \pm 1.46 in the control animals compared with $15.45 \pm 0.53$ and $15.62 \pm 0.34$ in rats in SH and UA groups, respectively. In addition, both RBC and HGB significantly increased following extract administration $(P<0.05)$.

All serological analyses were within normal ranges. However, conversion of serum glucose level (\%) in experimental rats to percent of controls indicated that glucose levels were significantly lower for male rats in the $\mathrm{SH}$ and UA groups $(79.83 \pm 11.10 \%$ and $76.09 \pm 9.67 \%$ of controls, respectively; $P<0.05)$ compared with control rats (Fig. 2A). Similarly, glucose levels were significant lower in female rats in SH and UA groups compared with control rats $(79.19 \pm 1.58 \%$ and $82.78 \pm 3.21 \%$ of controls, respectively; $P<0.01$ ) (Fig. 2B). Furthermore, ALP levels in male rats were significantly decreased in the $\mathrm{SH}$ and UA groups compared with the control group (332.06 \pm 27.64 and $303.06 \pm 10.31$, respectively, compared with 387.96 $\pm 30.90 ; P<0.05$ ), and TG and BUN levels were significantly decreased in the UA group compared with
A

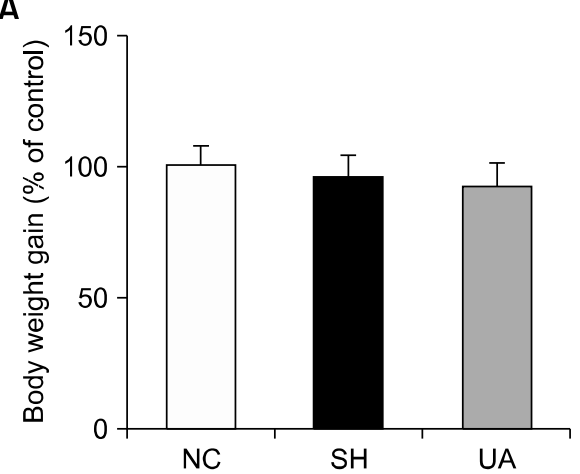

B

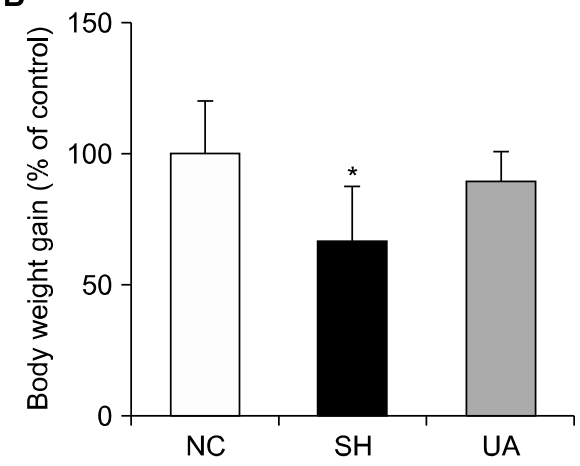

Fig. 1. Body weight gain rate of Sprague-Dawley rats treated with the extract for 14 days compared to the control group. (A) Male experimental group, (B) female experimental group. In the female experimental groups, the weight gain was significantly lower in the SH group administered with $2,000 \mathrm{mg} / \mathrm{kg}$ compared to the control group. The data were calculated and expressed as (the experimental group's final weight-initial weight)/(control group's later weight-initial weight) $\times 100$. Data are mean \pm SD $(n=5)$. ${ }^{*} P<0.05$ vs. normal control group. NC, normal control group; SH, Sargassum horneri administered group; UA, Ulva australis administered group.

Table 3. Food consumption of Sprague-Dawley rats orally administered ethanol extract of Sargassum horneri (SH) or U/va australis (UA) for 14 days

\begin{tabular}{|c|c|c|c|c|c|c|}
\hline \multirow{2}{*}{ Extract } & \multirow{2}{*}{ Sex } & \multirow{2}{*}{$\begin{array}{c}\text { Dose } \\
(\mathrm{mg} / \mathrm{kg} / \mathrm{d})\end{array}$} & \multicolumn{4}{|c|}{ Days after treatment } \\
\hline & & & 1 & 4 & 7 & 10 \\
\hline \multirow[t]{4}{*}{$\mathrm{SH}$} & Male & 0 & $22.7 \pm 0.4$ & $23.9 \pm 0.5$ & $23.5 \pm 0.7$ & $24.6 \pm 0.5$ \\
\hline & & 2,000 & $21.5 \pm 0.8$ & $23.0 \pm 0.8$ & $23.9 \pm 0.3$ & $23.3 \pm 0.4$ \\
\hline & Female & 0 & $19.7 \pm 2.4$ & $21.0 \pm 1.5$ & $20.4 \pm 1.1$ & $18.1 \pm 0.8$ \\
\hline & & 2,000 & $15.8 \pm 0.4$ & $14.9 \pm 0.2$ & $15.0 \pm 1.1$ & $15.9 \pm 1.2$ \\
\hline \multirow[t]{4}{*}{ UA } & Male & 0 & $22.7 \pm 0.4$ & $23.9 \pm 0.5$ & $23.5 \pm 0.7$ & $24.6 \pm 0.5$ \\
\hline & & 2,000 & $22.2 \pm 0.6$ & $23.0 \pm 0.2$ & $23.1 \pm 0.4$ & $23.6 \pm 0.3$ \\
\hline & Female & 0 & $19.7 \pm 2.4$ & $21.0 \pm 1.5$ & $20.4 \pm 1.1$ & $18.1 \pm 0.8$ \\
\hline & & 2,000 & $15.6 \pm 0.4$ & $16.5 \pm 0.2$ & $16.5 \pm 0.9$ & $15.6 \pm 0.5$ \\
\hline
\end{tabular}

Values were expressed as food consumption in gram/rat/d (mean \pm SD). 
Table 4. Absolute organ weights of Sprague-Dawley rats orally administered ethanol extracts of Sargassum horneri (SH) or U/va australis (UA) for 14 days

\begin{tabular}{|c|c|c|c|c|c|c|}
\hline \multirow{3}{*}{ Organ } & \multicolumn{3}{|c|}{ Male dose $(\mathrm{mg} / \mathrm{kg})$} & \multicolumn{3}{|c|}{ Female dose $(\mathrm{mg} / \mathrm{kg})$} \\
\hline & Control & $\mathrm{SH}$ & UA & Control & $\mathrm{SH}$ & UA \\
\hline & 0 & 2,000 & 2,000 & 0 & 2,000 & 2,000 \\
\hline Liver & $9.50 \pm 0.34$ & $9.45 \pm 1.16$ & $8.74 \pm 0.83$ & $6.69 \pm 0.47$ & $7.01 \pm 1.26$ & $7.18 \pm 0.84$ \\
\hline Spleen & $0.81 \pm 0.09$ & $0.78 \pm 0.13$ & $0.79 \pm 0.05$ & $0.58 \pm 0.12$ & $0.58 \pm 0.08$ & $0.57 \pm 0.04$ \\
\hline Kidney-left & $1.18 \pm 0.10$ & $1.11 \pm 0.11$ & $1.08 \pm 0.10$ & $0.74 \pm 0.09$ & $0.75 \pm 0.04$ & $0.81 \pm 0.15$ \\
\hline Kidney-right & $1.24 \pm 0.10$ & $1.21 \pm 0.10$ & $1.17 \pm 0.09$ & $0.77 \pm 0.11$ & $0.83 \pm 0.06$ & $0.83 \pm 0.18$ \\
\hline Heart & $1.13 \pm 0.21$ & $1.07 \pm 0.05$ & $1.07 \pm 0.10$ & $0.70 \pm 0.04$ & $0.72 \pm 0.07$ & $0.71 \pm 0.09$ \\
\hline Lung & $1.46 \pm 0.04$ & $1.57 \pm 0.17$ & $1.45 \pm 0.08$ & $1.17 \pm 0.19$ & $1.14 \pm 0.04$ & $1.17 \pm 0.12$ \\
\hline Thymus & $0.67 \pm 0.03$ & $0.64 \pm 0.13$ & $0.56 \pm 0.10$ & $0.52 \pm 0.07$ & $0.53 \pm 0.11$ & $0.43 \pm 0.03$ \\
\hline Testis-left & $1.49 \pm 0.15$ & $1.53 \pm 0.10$ & $1.51 \pm 0.13$ & - & - & - \\
\hline Testis-right & $1.54 \pm 0.13$ & $1.58 \pm 0.15$ & $1.56 \pm 0.11$ & - & - & - \\
\hline Prostate & $0.94 \pm 0.19$ & $0.87 \pm 0.17$ & $0.95 \pm 0.08$ & - & - & - \\
\hline Ovary-left & - & - & - & $0.11 \pm 0.07$ & $0.08 \pm 0.01$ & $0.09 \pm 0.01$ \\
\hline Ovary-right & - & - & - & $0.13 \pm 0.03$ & $0.10 \pm 0.02$ & $0.09 \pm 0.02$ \\
\hline Uterus & - & - & - & $0.60 \pm 0.19$ & $0.61 \pm 0.28$ & $0.54 \pm 0.13$ \\
\hline
\end{tabular}

Organ weight values were expressed in grams (mean \pm SD).

Table 5. Hematological parameters of Sprague-Dawley rats orally administered ethanol extracts of Sargassum horneri (SH) or Ulva australis (UA) for 14 days

\begin{tabular}{|c|c|c|c|c|c|c|}
\hline \multirow{3}{*}{ Parameter } & \multicolumn{3}{|c|}{ Male dose $(\mathrm{mg} / \mathrm{kg})$} & \multicolumn{3}{|c|}{ Female dose $(\mathrm{mg} / \mathrm{kg})$} \\
\hline & Control & $\mathrm{SH}$ & UA & Control & $\mathrm{SH}$ & UA \\
\hline & 0 & 2,000 & 2,000 & 0 & 2,000 & 2,000 \\
\hline WBC $\left(\times 10^{3} / \mu \mathrm{L}\right)$ & $7.81 \pm 0.88$ & $7.54 \pm 1.69$ & $7.35 \pm 0.75$ & $7.46 \pm 0.49$ & $7.62 \pm 0.43$ & $7.38 \pm 0.42$ \\
\hline $\mathrm{RBC}\left(\times 10^{6} / \mu \mathrm{L}\right)$ & $6.41 \pm 0.14$ & $6.38 \pm 0.28$ & $6.36 \pm 0.08$ & $6.32 \pm 0.68$ & $7.77 \pm 0.46^{*}$ & $7.48 \pm 0.46^{*}$ \\
\hline $\mathrm{HGB}(\mathrm{g} / \mathrm{dL})$ & $14.49 \pm 0.67$ & $13.91 \pm 0.35$ & $14.21 \pm 0.23$ & $13.44 \pm 1.46$ & $15.45 \pm 053^{*}$ & $15.62 \pm 0.34^{*}$ \\
\hline HCT (\%) & $40.82 \pm 0.66$ & $41.90 \pm 3.45$ & $40.68 \pm 0.75$ & $37.08 \pm 4.21$ & $39.95 \pm 3.41$ & $38.12 \pm 1.47$ \\
\hline MCV (fL) & $63.94 \pm 0.66$ & $63.86 \pm 1.31$ & $63.98 \pm 0.69$ & $58.66 \pm 0.78$ & $58.35 \pm 0.92$ & $58.37 \pm 1.17$ \\
\hline $\mathrm{MCH}(\mathrm{pg})$ & $22.38 \pm 0.43$ & $22.08 \pm 0.84$ & $22.32 \pm 0.28$ & $21.28 \pm 0.31$ & $19.92 \pm 0.56$ & $20.96 \pm 0.83$ \\
\hline $\mathrm{MCHC}(\mathrm{g} / \mathrm{dL})$ & $35.02 \pm 0.77$ & $34.52 \pm 1.01$ & $34.96 \pm 0.64$ & $36.26 \pm 0.34$ & $34.13 \pm 0.54$ & $35.29 \pm 1.28$ \\
\hline PLT $\left(\times 10^{3} / \mu \mathrm{L}\right)$ & $637.68 \pm 34.33$ & $699.78 \pm 31.82$ & $680.28 \pm 32.84$ & $672.80 \pm 31.35$ & $640.65 \pm 32.36$ & $672.83 \pm 27.21$ \\
\hline
\end{tabular}

Values were expressed as means \pm SD $(n=5)$.

Significant differences at ${ }^{*} P<0.05$.

WBC, white blood cell; RBC, red blood cell; HGB, hemoglobin; $H C T$, hematocrit; MCV, mean red blood cell volume; $M C H$, mean corpuscular hemoglobin; $\mathrm{MCHC}$, mean corpuscular hemoglobin concentration; PLT, platelet.

A

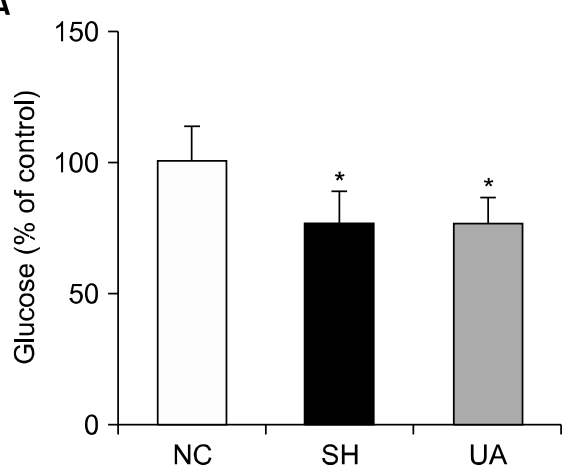

B

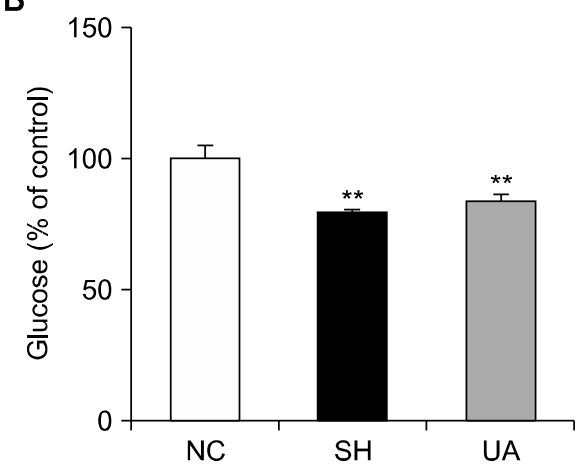

Fig. 2. Serum glucose levels of Sprague-Dawley Rats treated with the extract for 14 days compared to the control group. (A) male experimental group, (B) female experimental group. Serum glucose levels (\%) were significantly decreased in both male and female experimental groups compared to the control group. The data were calculated and expressed as the experimental group glucose level/control group glucose level $\times 100$. Data are mean $\pm \mathrm{SD}(\mathrm{n}=5)$. ${ }^{*} P<0.05$ and ${ }^{* *} P<0.01$ vs. normal control group. NC, normal control group; SH, Sargassum horneri administered group; UA, U/va australis administered group. 
Table 6. Serum biochemical parameters of Sprague-Dawley rats orally administered ethanol extracts of Sargassum horneri (SH) or Ulva australis (UA) for 14 days

\begin{tabular}{|c|c|c|c|c|c|c|}
\hline \multirow{3}{*}{ Parameter } & \multicolumn{3}{|c|}{ Male dose $(\mathrm{mg} / \mathrm{kg})$} & \multicolumn{3}{|c|}{ Female dose $(\mathrm{mg} / \mathrm{kg})$} \\
\hline & Control & $\mathrm{SH}$ & UA & Control & $\mathrm{SH}$ & UA \\
\hline & 0 & 2,000 & 2,000 & 0 & 2,000 & 2,000 \\
\hline TP (g/dL) & $5.94 \pm 0.21$ & $6.22 \pm 0.22$ & $5.89 \pm 0.31$ & $5.51 \pm 0.29$ & $5.85 \pm 0.35$ & $5.80 \pm 0.28$ \\
\hline ALB (g/dL) & $3.53 \pm 0.12$ & $3.69 \pm 0.15$ & $3.56 \pm 0.22$ & $3.32 \pm 0.20$ & $3.43 \pm 0.14$ & $3.44 \pm 0.12$ \\
\hline AST (U/L) & $121.26 \pm 7.55$ & $128.27 \pm 7.35$ & $122.66 \pm 5.70$ & $108.68 \pm 17.22$ & $128.25 \pm 7.44^{*}$ & $108.50 \pm 7.45$ \\
\hline ALT (U/L) & $53.20 \pm 4.21$ & $49.80 \pm 3.35$ & $48.20 \pm 4.66$ & $38.00 \pm 7.78$ & $40.00 \pm 5.89$ & $38.00 \pm 6.28$ \\
\hline GGT (U/L) & $2.18 \pm 0.59$ & $1.37 \pm 0.81$ & $1.92 \pm 0.66$ & $2.35 \pm 0.66$ & $2.33 \pm 0.76$ & $2.19 \pm 1.16$ \\
\hline ALP (U/L) & $387.96 \pm 30.90$ & $332.06 \pm 27.64^{*}$ & $303.06 \pm 10.31^{*}$ & $180.32 \pm 20.70$ & $160.20 \pm 14.02$ & $158.28 \pm 14.75$ \\
\hline GLU (mg/dL) & $118.45 \pm 16.60$ & $90.73 \pm 10.75^{*}$ & $90.12 \pm 12.80^{*}$ & $188.46 \pm 9.22$ & $148.25 \pm 2.86^{* *}$ & $156.00 \pm 6.77^{* *}$ \\
\hline $\mathrm{CHO}(\mathrm{mg} / \mathrm{dL})$ & $79.40 \pm 5.89$ & $81.82 \pm 5.44$ & $72.19 \pm 4.28$ & $78.70 \pm 7.28$ & $86.38 \pm 5.84$ & $86.67 \pm 4.26$ \\
\hline TG (mg/dL) & $50.93 \pm 11.66$ & $45.22 \pm 5.19$ & $35.97 \pm 5.11^{*}$ & $33.49 \pm 3.02$ & $43.69 \pm 2.42^{* *}$ & $34.10 \pm 4.92$ \\
\hline TB (mg/dL) & $0.83 \pm 0.04$ & $0.84 \pm 0.02$ & $0.79 \pm 0.07$ & $0.79 \pm 0.06$ & $0.75 \pm 0.44$ & $0.78 \pm 0.06$ \\
\hline $\mathrm{HDL}-\mathrm{C}(\mathrm{mg} / \mathrm{dL})$ & $49.61 \pm 6.89$ & $54.63 \pm 5.47$ & $51.56 \pm 3.54$ & $57.68 \pm 7.59$ & $70.43 \pm 4.80^{*}$ & $62.60 \pm 4.37$ \\
\hline Urea (mg/dL) & $1.49 \pm 0.37$ & $1.77 \pm 0.26$ & $1.26 \pm 0.18$ & $1.13 \pm 0.38$ & $1.18 \pm 0.18$ & $1.07 \pm 0.24$ \\
\hline BUN (mg/dL) & $24.49 \pm 1.97$ & $22.74 \pm 1.72$ & $19.47 \pm 3.47^{*}$ & $18.44 \pm 1.71$ & $15.09 \pm 1.44$ & $16.43 \pm 1.95$ \\
\hline
\end{tabular}

Values were expressed as means $\pm S D(n=5)$.

Significant differences at ${ }^{*} P<0.05$ and ${ }^{* *} P<0.01$ vs. the control group.

TP, total protein; ALB, albumin; AST, aspartate aminotransferase; ALT, alanine transaminase; GGT, gamma-glutamyl transferase; ALP, alkaline phosphatase; GLU, glucose; $\mathrm{CHO}$, total cholesterol; TG, triglyceride; TB, total bilirubin; HDL-C, high-density lipoprotein cholesterol; BUN, blood urea nitrogen.

the control group $(P<0.05)$. In addition, female rats treated with SH showed significantly increased AST $(P<$ $0.05)$, TG $(P<0.01)$, and HDL-C $(P<0.05)$ levels compared with control rats (Table 6).

\section{DISCUSSION}

This study was designed to support a method for efficient recycling of $\mathrm{SH}$ and UA waste, which cause substantial organic pollution along the Jeju coast. SH and UA extracts were orally administered to laboratory rats for 14 days, following drug toxicity testing criteria (No. 2009116).

During extract administration, no rats died. Furthermore, extract administration did not induce salivation, which frequently occurs during drug oral drug delivery (Han et al., 2004; Yoon et al., 2006; Kim et al., 2012), and there were no signs of altered motility or toxicity.

Body weight gain was recorded as a gross measure of toxicity, and was lower for rats treated with $\mathrm{SH}$ and UA compared with control rats (Bailey et al., 2004). SH induced lower body weight gain than UA in female rats, whereas UA induced lower body weight gain than $\mathrm{SH}$ in male rats. Addressing gender differences has recently emerged as a priority in several medical fields, including metabolic diseases (Rich-Edwards et al., 2018). Differences in metabolism, gene expression, anatomy, behavior, stress responses, and longevity may vary between genders and may influence carbohydrate and lipid metabolism (Tower et al., 2020; Tramunt et al., 2020). Such differences affect many aspects of metabolism and related diseases, reflecting differences in male and female sex hormones (Link and Reue, 2017). Responses in female and male animals differed dependent on these factors.

Feed intake was lower in female rats administered $\mathrm{SH}$ and UA compared with control rats, although the differences were not significant. However, no differences were observed between control and experimental male animals. In a previous study, $\mathrm{SH}$ powder was added to white shrimp (Litopenaeus vannamei) feed; although no significant differences in feed intake were found, the growth rate of the shrimp was lower (Eom et al., 2020). The weight loss observed in the current study is likely an effect extract administration since $\mathrm{SH}$ exhibits has antiobesity activity (Murakami et al., 2021). Body weight of rats following UA extract administration was lower than that of control rats. These results are consistent with chronic toxicity, which has previously been reported for UA (Qi et al., 2013). Indeed, Capsosiphon fulvescens in the family Ulvaceae induces significant weight loss when combined with a high cholesterol diet (Kwon and Nam, 2006). Our results suggest that UA may be a functional substance to aid weight loss.

Changes in the weights of major organs are important indicators of toxicity (Bailey et al., 2004). Compared with control rats, rats administered $\mathrm{SH}$ and UA extracts did not exhibit any significant difference in organ weight. and the two substances appear to be non-toxic. However, organ weights tended to be higher than those recorded in historical data (Kang et al., 2001). However, in the previous study, experimental animals were seven weeks old 
when organ weights were measured, whereas in the current study organ weights were measured when rats were eight weeks of age. Therefore, higher weights were likely due to this difference in age.

Hematological examination showed that RBC and HGB levels significantly increased in female rats administered $\mathrm{SH}$ and UA extracts, but no significant changes were observed for $\mathrm{MCV}, \mathrm{MCH}$, and $\mathrm{MCHC}$. Compared with data from the previous study values, no abnormal measurements were observed after extract administration, and all values were within normal ranges (Wolford et al., 1986).

Serological tests were conducted to confirm the presence or absence of liver and kidney abnormalities induced by $\mathrm{SH}$ and UA and the health statuses of the experimental rats. Results showed significantly reduced ALP and glucose levels in male rats following extract administration. In rats administered urea, TG and BUN values decreased significantly but changed remained within the normal range (Kang et al., 2004). In females, glucose were significantly decreased in both extract groups, and AST, TG, and HDL-C levels were significantly increased following administration of $\mathrm{SH}$ compared with control rats; however, the values remained within normal ranges. Results of previous studies suggest that Sargassum sp. and Ulva sp. can lower serum glucose levels (Kang et al., 2012; Kim and Byun, 2018), therefore the serum glucose reducing effect shown in this study was probably induced by the experimental extracts.

Overall, rats administered algal extracts showed similar responses as rats administered distilled water as a control. However, weight gain after extract administration was low, and serum biochemistry analyses showed significantly lowered blood sugar levels. However, these effects are not expected to result in notable toxicity. Therefore, algal waste may be used safely as functional additives for consumption by both animals and humans. However, additional studies are needed to explore weight loss and hypoglycemia in models of obesity and diabetes.

\section{ACKNOWLEDGEMENTS}

This study was supported by the Industrialization of Organic Resources in Jeju Special Self-Governing Province and Brain Busan 21 Plus Project.

\section{AUTHOR DISCLOSURE STATEMENT}

The authors declare no conflict of interest.

\section{REFERENCES}

Ahn MS, Kim HJ, Seo MS. A study on the antioxidative and antimicrobial activities of the Citrus unshju peel extracts. Korean J Food Culture. 2007. 22:454-461.

Bailey SA, Zidell RH, Perry RW. Relationships between organ weight and body/brain weight in the rat: what is the best analytical endpoint?. Toxicol Pathol. 2004. 32:448-466.

Byeon SY, Oh HJ, Kim S, Yun SH, Kang JH, Park SR, et al. The origin and population genetic structure of the 'golden tide' seaweeds, Sargassum horneri, in Korean waters. Sci Rep. 2019. 9: 7757. https://doi.org/10.1038/s41598-019-44170-x

Cardoso SM, Pereira OR, Seca AM, Pinto DC, Silva AM. Seaweeds as preventive agents for cardiovascular diseases: from nutrients to functional foods. Mar Drugs. 2015. 13:6838-6865.

Chi Y, Zhang M, Wang X, Fu X, Guan H, Wang P. Ulvan lyase assisted structural characterization of ulvan from Ulva pertusa and its antiviral activity against vesicular stomatitis virus. Int J Biol Macromol. 2020. 157:75-82.

Choi HJ, Kil JH, Bak SS, Kong CS, Park KY, Seo Y, et al. Inhibitory effects of solvent extracts from seven brown algae on mutagenicity and growth of human cancer cells. J Life Sci. 2006. 16: 1080-1086.

Dias MKHM, Madusanka DMD, Han EJ, Kim MJ, Jeon YJ, Kim HS, et al. (-)-Loliolide isolated from Sargassum horneri protects against fine dust-induced oxidative stress in human keratinocytes. Antioxidants. 2020. 9:474. https://doi.org/10.3390/ antiox 9060474

Eom G, Shin J, Lim SJ, Lee KT, Han YS, Lee KJ. Evaluation of dietary supplementation of Sargassum horneri for pacific white shrimp Litopenaeus vannamei. Korean J Fish Aquat Sci. 2020. 53: 909-917.

Han SC, Suh KH, Cha SW, Suh JE. Single oral dose toxicity of a new salt of sibutramine, sibutramine mesilate hemihydrate, in rats and beagle dogs. Korean J Lab Anim Sci. 2004. 20:409418.

Hong JK, Bong MH, Park JC, Moon HK, Kim DW, Lee SC, et al. Antioxidant and immunomodulatory effects of Ulva pertusa kjellman on broiler chickens. J Anim Sci Technol. 2011. 53:419428.

Kang BH, Kim IH, Kim YB, Kim YH, Lee HS, Ha CS. Reference values of organ weights in Sprague-Dawley rats. Korean J Vet Pathol. 2001. 5:39-42.

Kang BH, Kim YB, Lee HS, Kim YH, Im WJ, Ha CS. Background data on hematology, blood biochemistry and organ weights for 2 weeks and 4 weeks repeated-dose toxicity studies using Sprague-Dawley (SD) rats. Korean J Lab Anim Sci. 2004. 20: 134-140.

Kang SH, Cho EK, Cho YJ. $\alpha$-Glucosidase inhibitory effects for solvent fractions from methanol extracts of Sargassum fulvellum and its antioxidant and alcohol-metabolizing activities. J Life Sci. 2012. 22:1420-1427.

Kim DS, Sung NY, Park SY, Kim G, Eom J, Yoo JG, et al. Immunomodulating activity of Sargassum horneri extracts in RAW264.7 macrophages. J Nutr Health. 2018a. 51:507-514.

Kim HG, Lim HA, Kim SY, Kang SS, Lee HY, Yun PY. Development of functional hanji added citrus peel(I) - Hanji added Korean citrus peel - . J Korea TAPPI. 2007. 39:38-47.

Kim HM, Jo J, Park C, Choi BJ, Lee HG, Kim KY. Epibionts associated with floating Sargassum horneri in the Korea Strait. Algae. 2019. 34:303-313.

Kim HS, Han EJ, Fernando IPS, Sanjeewa KKA, Jayawardena TU, Kim HJ, et al. Anti-allergy effect of mojabanchromanol isolated from Sargassum horneri in bone marrow-derived cultured mast cells. Algal Res. 2020. 48:101898. https://doi.org/10.1016/ j.algal.2020.101898 
Kim JA, Karadeniz F, Ahn BN, Kwon MS, Mun OJ, Kim M, et al. Sargassum sp. attenuates oxidative stress and suppresses lipid accumulation in vitro. J Life Sci. 2014. 24:274-283.

Kim JS, Kim BG, Kwak HS. Simultaneous application of chemicals and temperature for the effective control of trouble seaweed Ulva australis. Weed Turf Sci. 2018b. 7:35-45.

Kim NG. Effects of temperature, photon irradiance, and photoperiod on the growth of embryos of Sargassum horneri in laboratory culture. Kor J Fish Aquat Sci. 2015. 48:76-81.

Kim SK, Byun HG. Production and biological applications for marine proteins and peptides - an overview. Food Science and Industry. 2018. 51:278-301.

Kim SR, Lee JW, Lim SY, Jung YS, Choi HY, Kim JD. Rat single oral dose toxicity test of Armeniacae semen (including endocarp). Korean J Orient Int Med. 2012. 33:145-159.

Kim YM, Kim DS, Choi YS. Anticoagulant activities of brown seaweed extracts in Korea. Korean J Food Sci Technol. 2004. 36: 1008-1013.

Kwon DH, Choi YH, Kim BW, Hwang HJ. Effects of ethanol extract of Sargassum horneri on adipocyte differentiation and adipogenesis in 3T3-L1 preadipocytes. J Life Sci. 2019. 29:209214.

Kwon MJ, Nam TJ. Effects of mesangi (Capsosiphon fulvecens) powder on lipid metabolism in high cholesterol fed rats. J Korean Soc Food Sci Nutr. 2006. 35:530-535.

Lin AP, Wang C, Pan GH, Song LY, Gao S, Xie XJ, et al. Diluted seawater promoted the green tide of Ulva prolifera (Chlorophyta, Ulvales). Phycol Res. 2011. 59:295-304.

Link JC, Reue K. Genetic basis for sex differences in obesity and lipid metabolism. Annu Rev Nutr. 2017. 37:225-245.

Liu L, Heinrich M, Myers S, Dworjanyn SA. Towards a better understanding of medicinal uses of the brown seaweed Sargassum in Traditional Chinese Medicine: a phytochemical and pharmacological review. J Ethnopharmacol. 2012. 142:591-619.

Matsumura Y. Nutrition trends in Japan. Asia Pac J Clin Nutr. 2001. 10:S40-S47.

Murakami S, Hirazawa C, Ohya T, Yoshikawa R, Mizutani T, Ma $\mathrm{N}$, et al. The edible brown seaweed Sargassum horneri (Turner) C. Agardh ameliorates high-fat diet-induced obesity, diabetes, and hepatic steatosis in mice. Nutrients. 2021. 13:551. https:// doi.org/10.3390/nu13020551

Patel S. Therapeutic importance of sulfated polysaccharides from seaweeds: updating the recent findings. 3 Biotech. 2012. 2:171185.

Preeprame S, Hayashi K, Lee JB, Sankawa U, Hayashi T. A novel antivirally active fucan sulfate derived from an edible brown alga, Sargassum horneri. Chem Pharm Bull. 2001. 49:484-485.

Qi H, Huang L, Liu X, Liu D, Zhang Q, Liu S. Antihyperlipidemic activity of high sulfate content derivative of polysaccharide extracted from Ulva pertusa (Chlorophyta). Carbohydr Polym. 2012. 87:1637-1640.

Qi H, Liu X, Wang K, Liu D, Huang L, Liu S, et al. Subchronic toxicity study of ulvan from Ulva pertusa (Chlorophyta) in Wistar rats. Food Chem Toxicol. 2013. 62:573-578.

Rich-Edwards JW, Kaiser UB, Chen GL, Manson JE, Goldstein JM. Sex and gender differences research design for basic, clinical, and population studies: essentials for investigators. Endocr Rev. 2018. 39:424-439.

Song L, Chen X, Liu X, Zhang F, Hu L, Yue Y, et al. Characterization and comparison of the structural features, immune-modulatory and anti-avian influenza virus activities conferred by three algal sulfated polysaccharides. Mar Drugs. 2015. 14:4. https://doi.org/10.3390/md14010004

Tower J, Pomatto LCD, Davies KJA. Sex differences in the response to oxidative and proteolytic stress. Redox Biol. 2020. 31:101488. https://doi.org/10.1016/j.redox.2020.101488

Tramunt B, Smati S, Grandgeorge N, Lenfant F, Arnal JF, Montagner A, et al. Sex differences in metabolic regulation and diabetes susceptibility. Diabetologia. 2020. 63:453-461.

Wolford ST, Schroer RA, Gohs FX, Gallo PP, Brodeck M, Falk HB, et al. Reference range data base for serum chemistry and hematology values in laboratory animals. J Toxicol Environ Health. 1986. 18:161-188.

Yoon SW, Park JW, Kim KS, Jung HS, Choi WC. The study on the safety and case series of the acute lymphocytic leukemia using Rhus verniciflua stokes extract (Nexia). J Korean Tradit Oncol. 2006. 11:1-21.

Zhang J, Huo Y, Yu K, Chen Q, He Q, Han W, et al. Growth characteristics and reproductive capability of green tide algae in Rudong coast, China. J Appl Phycol. 2013. 25:795-803. 